\title{
Influencia de factores ambientales y biométricos en la capacidad de nado del barbo ibérico (Luciobarbus bocagei Steindachner, 1864), un ciprínido potamódromo endémico de la Península Ibérica
}

\author{
J. Ruiz-Legazpi ${ }^{1, *}$, F.J. Sanz-Ronda ${ }^{1}$, F.J. Bravo-Córdoba ${ }^{1}$, J.F. Fuentes-Pérez ${ }^{1,2}$ y \\ T. Castro-Santos ${ }^{3}$
}

${ }^{1}$ ETSIIA, Universidad de Valladolid, UD de Hidráulica e Hidrología, Avda. de Madrid, 57, 34004 Palencia, España.

2 Centre for Biorobotics, Tallinn University of Technology, Akadeemia tee 15A-111, 12618 Tallinn, Estonia.

3 S.O. Conte Anadromous Fish Research Center, USGS-LSC, P.O. Box 796, One Migratory Way, Turners Falls, MA 01376, EE.UU.

* Corresponding author: jorge.ruiz.legazpi@iaf.uva.es

Received: 05/12/17

Accepted: 18/04/18

\section{RESUMEN}

Influencia de factores ambientales y biométricos en la capacidad de nado del barbo ibérico (Luciobarbus bocagei Steindachner, 1865), un ciprínido potamódromo endémico de la Península Ibérica

El presente trabajo analiza la capacidad voluntaria de nado del barbo ibérico (Luciobarbus bocagei Steindachner, 1864) en un canal abierto durante su época de migración, relacionándola con factores ambientales y biométricos. La temperatura del agua, la velocidad de flujo y la longitud del pez fueron los factores de mayor importancia que condicionaron la velocidad de nado de los barbos y su tiempo de fatiga. Dentro del rango de valores estudiado, el barbo ibérico pudo mantener velocidades de nado en sprint (> $15 \mathrm{BL} / \mathrm{s}$ ) durante 3-10 s, y de 17-117 s en el modo de natación prolongada (7-15 BL/s). Los resultados aportados pueden ser empleados como una herramienta útil para la gestión de sus poblaciones, principalmente para el diseño de pasos para peces.

Palabras clave: Barbo ibérico, capacidad nado, tiempo fatiga, análisis supervivencia, temperatura, velocidad, ciprínidos, Península Ibérica

\section{ABSTRACT}

Influence of environmental and biometric factors on the swimming capacity of the Iberian barbel (Luciobarbus bocagei Steindachner, 1864), an endemic potamodromous cyprinid of the Iberian Peninsula

This paper analyzes the volitional swimming capacity of the Iberian barbel (Luciobarbus bocagei Steindachner, 1864) in an open flume during its migration period, in relation to environmental and biometric factors. Water temperature, flow velocity and fish length were the most important factors which affected the swimming speed of barbels and their fatigue time. Within the range of values studied, the Iberian barbel was able to maintain sprint swim speeds (>15 BL/s) for 3-10 s, and 17-117 s in prolonged swim mode $(7-15 \mathrm{BL} / \mathrm{s})$. The results can be used as a tool for the management of barbel populations, mainly in the design of fishways.

Key words: Iberian barbel, swimming capacity, fatigue time, survival analysis, temperature, velocity, cyprinids, Iberian Peninsula 


\section{INTRODUCCIÓN}

El aprovechamiento de cursos fluviales en el desarrollo de las actividades humanas tiene un impacto en estos ecosistemas, fundamentalmente debido a la creación de barreras que alteran su conectividad longitudinal, causando, entre otros impactos, la fragmentación del hábitat (Cooke $e t$ al., 2012b). Los peces migradores son los más afectados por esta problemática (Lucas \& Baras, 2001), que puede bloquear o retrasar sus movimientos migratorios, tanto hacia aguas arriba como aguas abajo (Castro-Santos \& Haro, 2003), reducir sus hábitats y amenazar la viabilidad de sus poblaciones, llegando incluso a su extinción (Lucas \& Frear, 1997; Morita \& Yamamoto, 2002; Tudorache et al., 2008; Romão et al., 2012). Algunas barreras son infranqueables (e.g. grandes presas), mientras que otras exigen un esfuerzo de natación importante para ser superadas (e.g. pequeñas presas, estaciones de aforo o algunos pasos piscícolas), pudiendo llevar a los peces al límite de sus capacidades fisiológicas (Haro et al., 2004).

En el ámbito ibérico, el grado de alteración antropogénica en los ecosistemas fluviales es muy elevado, por ejemplo, en la parte española de la cuenca del Duero, se estima que existe una barrera aproximadamente cada $2.7 \mathrm{~km}$ de río (CHD, 2015).

La capacidad de nado de los peces es un factor crucial que determina el nivel de impacto de las obras hidráulicas y su conocimiento permite proponer soluciones para paliar los efectos de éstas (Sanz-Ronda et al., 2015). Habitualmente, la capacidad de nado se ha venido cuantificando mediante el método propuesto por Brett (1964), usando respirómetros y estimando la velocidad crítica de nado $\left(\mathrm{U}_{\text {crit }}\right)$ en condiciones forzadas (Peake, 2008a).

$\mathrm{U}_{\text {crit }}$ es una categoría especial de velocidad prolongada (Beamish, 1978; Hammer, 1995), que se ha considerado en el diseño de pasos para peces y caños de drenaje (Mateus et al., 2008; Santos et al., 2012). $\mathrm{U}_{\text {crit }}$ ha recibido críticas por su ambigüedad ecológica, ya que puede subestimar la capacidad de nado (Tudorache et al., 2007; Peake, 2008b; Castro-Santos et al., 2013), derivando en diseños excesivamente conservado- res que pueden llegar a imposibilitar el ascenso en lugar de facilitarlo (Castro-Santos, 2004).

Un sistema que permite determinar la capacidad de natación de los peces en condiciones más naturales son los canales de nado (Videler \& Wardle, 1991; Haro et al., 2004). Estos sistemas se basan en la natación voluntaria, a menudo contra velocidades de flujo elevadas, que reflejan mejor las barreras de velocidad (Haro et al., 2004) y que facilitan a los individuos desarrollar sus capacidades máximas, al permitir la natación en modo sprint (Weaver, 1963; Castro-Santos, 2005). Los canales de nado también posibilitan cuantificar la capacidad de nado de una manera más completa, definiéndola en base a tres métricas: distancia máxima que es capaz de recorrer un individuo, tiempo de fatiga que tarda en recorrer dicha distancia y velocidad de nado (absolutarelativa) que puede desarrollar al recorrerla.

La capacidad de nado de los peces está condicionada por parámetros ambientales y biológicos. De todos ellos, se suelen considerar como los más importantes la temperatura del agua, la velocidad del flujo y el tamaño de los peces (Wardle, 1975; Videler, 1993; Lucas \& Baras, 2001). La temperatura del agua afecta al metabolismo (Plaut, 2001), la velocidad de flujo determina el modo de nado (Pavlov et al., 2002) y el tamaño de los individuos influye en su fortaleza y resistencia al movimiento (Boily \& Magnan, 2002).

La información sobre la capacidad de nado de los ciprínidos potamódromos ibéricos está en aumento. Durante la última década se han desarrollado estudios interesantes sobre ciprínidos, ayudando a comprender mejor su comportamiento natatorio (Mateus et al., 2008; Romão et al., 2012; Silva et al., 2012; Branco et al., 2013; Alexandre et al., 2014; Sanz-Ronda et al., 2015; Alexandre et al., 2016; Bravo-Córdoba et al., 2017). Dentro de este grupo destacan los barbos del género Luciobarbus (Tabla S1 disponible en http://www.limnetica.net/es/limnetica; Doadrio et al., 2011; Kottelat \& Freyhof, 2007).

Por lo tanto, el objetivo de este trabajo es determinar la capacidad de nado voluntario del barbo ibérico (Luciobarbus bocagei) y cómo influyen en ella factores ambientales (temperatura del agua y velocidad del flujo) y biométricos (longitud y masa). Se ha considerado esta especie 


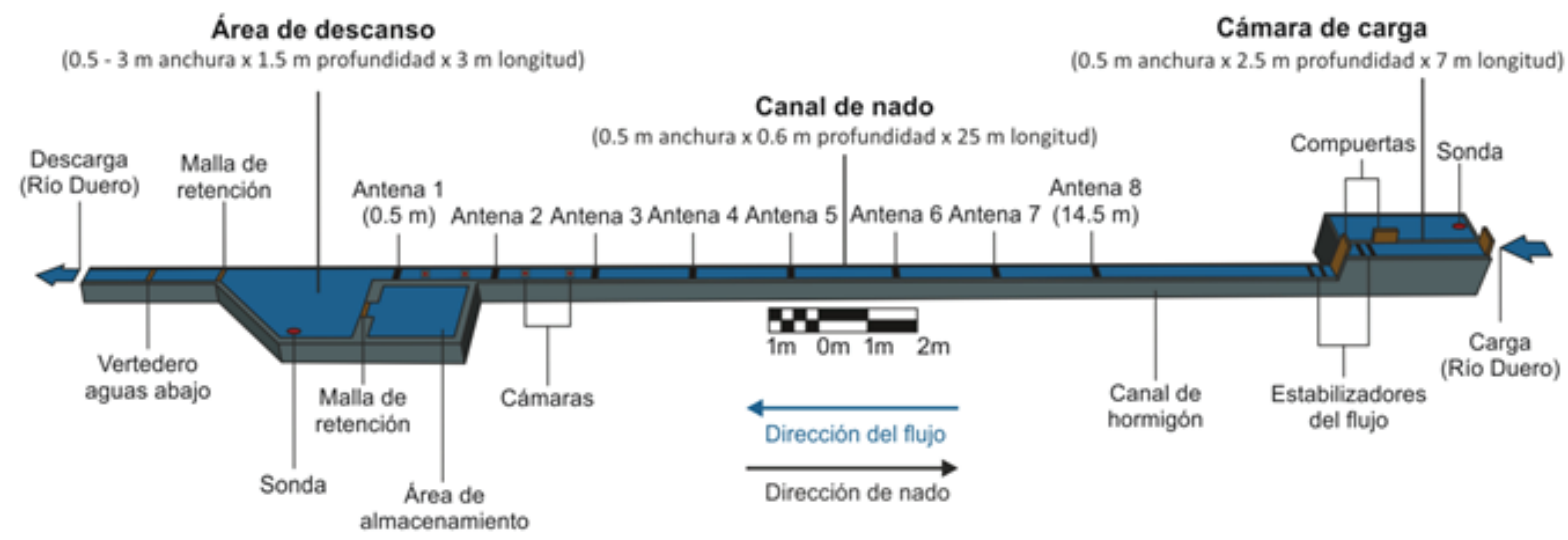

Figura 1. Esquema del canal de nado utilizado para la experimentación con los tres módulos principales y los sistemas de control de peces (PIT y cámaras) y de control hidráulico (compuertas, vertederos y sondas). Open-channel flume scheme used for experimentation. Three main modules are shown, as well as fish monitoring (PIT and video cameras) and hydraulic control (gates, spillway, and depth loggers) systems.

debido a que es uno de los ciprínidos potamódromos endémicos de mayor distribución y abundancia en la Península Ibérica y dada su similitud morfológica y biológica con sus congéneres ibéricos (Doadrio et al., 2011). Esta información puede ser útil para desarrollar directrices técnicas y científicas adecuadas en el diseño de pasos para peces, que repercutan positivamente en la conservación de sus poblaciones.

\section{MATERIALES Y MÉTODOS}

\section{Canal de nado}

El estudio se ha llevado a cabo en un canal de nado ubicado en las instalaciones de la central hidroeléctrica de Vadocondes (Burgos, España), en el río Duero (ETRS89 41 38' 16.05" N; $3^{\circ} 34^{\prime}$ $\left.17.37^{\prime \prime} \mathrm{W}\right)$. El canal está construido en obra con hormigón armado, tiene pendiente cero y consta de tres módulos (Fig. 1): cámara de carga, canal de nado y área de descanso. Un sistema de compuertas regula el caudal circulante, fijando la velocidad y profundidad del flujo.

\section{Instrumentación}

La distancia de natación recorrida por cada pez dentro del canal y el tiempo que emplearon para ello fueron determinados por la combinación de dos sistemas: telemetría y videocámaras. El equipo de telemetría está basado en transmisores electromagnéticos conocidos como PIT (Passive Integrated Transponder), y consta de un conjunto de ocho antenas equidistantes $2 \mathrm{~m}$ (la primera a $0.5 \mathrm{~m}$ del inicio del canal y la última a $14.5 \mathrm{~m}$ ) y 2 lectores sincronizados (Multi-Antenna HDX Reader -wired synchronization Master/Slave with acknowledgement-, Oregon-RFID, Oregon, EE.UU.). El sistema de videocámaras (Sony 420TVL-CCD 36-IR-LED Day\&Night CCTV; 15-fps, Sony, Tokio, Japón) consiste en cuatro cámaras cenitales entre las antenas 2 y 4 , con un área de grabación individual de $0.8 \mathrm{~m}$ de largo y $0.5 \mathrm{~m}$ de ancho, que complementan la medición de la distancia en los espacios entre antenas.

La distancia máxima recorrida $\left(\mathrm{D}_{\max }\right)$ se estima como:

$$
D_{\text {max }}=0.5+D_{j}+D_{j+}
$$

donde 0.5 es la distancia $(\mathrm{m})$ entre el inicio del canal y la antena $1, D_{j}$ es la distancia (m) entre la última antena donde se detectó el pez (antena j) y la antena $1, \mathrm{y} \mathrm{D}_{\mathrm{j}+}$ es la distancia $(\mathrm{m})$ recorrida más allá de la última antena observada (antena j). 
El tiempo de fatiga $\left(T_{f}\right)$ puede estimarse mediante la expresión:

$$
\mathrm{T}_{\mathrm{f}}=\mathrm{T}_{0.5}+\mathrm{T}_{\mathrm{j}}+\mathrm{T}_{\mathrm{j}+}
$$

donde $\mathrm{T}_{0.5}$ se refiere al tiempo (s) empleado en ascender los primeros $0.5 \mathrm{~m}$ del canal (calculado como $1 / 4$ del tiempo transcurrido entre las antenas 1 y 2), $T_{j}$ es el tiempo (s) necesario para recorrer la distancia $D_{j}, y T_{j+}$ es el tiempo (s) de ascenso por encima de la última antena (antena j).

A partir de las ecuaciones 1 y 2 se define la velocidad de nado relativa $\left(\mathrm{U}_{\mathrm{g}}\right)$ como:

$$
\mathrm{U}_{\mathrm{g}}=\frac{\mathrm{D}_{\max }}{\mathrm{T}_{\mathrm{f}}}
$$

Finalmente, se define la velocidad absoluta de nado $\left(\mathrm{U}_{\mathrm{s}}\right)$ como:

$$
\mathrm{U}_{\mathrm{s}}=\mathrm{U}_{\mathrm{g}}+\mathrm{U}_{\mathrm{f}}
$$

donde $\mathrm{U}_{\mathrm{f}}$ es la velocidad de flujo $(\mathrm{m} / \mathrm{s})$, definida como la velocidad media contra la cual cada pez nada (Sanz-Ronda et al., 2015). Las velocidades de nado se expresan, generalmente en cuerpos por segundo $(\mathrm{BL} / \mathrm{s})$, métrica que relativiza la velocidad de nado en función del tamaño del pez, facilitando la comparación entre individuos.

Durante la experimentación, se realizó una monitorización continua del canal: profundidad cada minuto (sonda Orfeo-Mini, OTT Hydromet $\mathrm{GmbH}$, Kempten, Alemania; Precisión: $0.002 \mathrm{~m}$ ), velocidad del flujo cada 30 minutos (velocímetro Modelo 2100, Swoffer Instruments Inc., Washington, EE.UU. Precisión: $0.01 \mathrm{~m} / \mathrm{s}$ ) y parámetros fisicoquímicos cada hora, cuyos valores se muestran en la Tabla S2 (disponible en http://www.limnetica. net/es/limnetica).

\section{Muestra de peces}

Los peces fueron capturados mediante pesca eléctrica (ERREKA III, DC, 200 V, Acuitec S.L., Gipuzkoa, España) en el río Duero, en un radio de $2 \mathrm{~km}$ del canal. Las capturas se efectuaron en dos momentos (25-26 de mayo de 2012 y 5-6 de mayo de 2013), ambos dentro del periodo habitual de freza en esta región (mayo-junio) (Doadrio et al.,

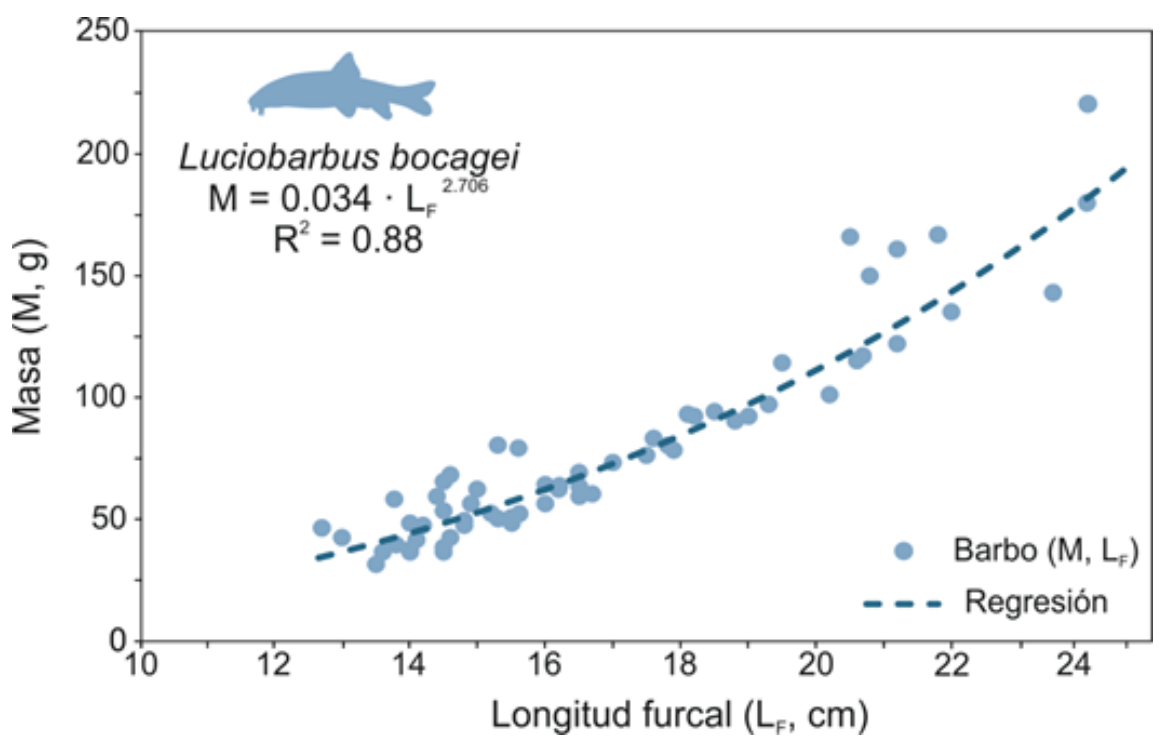

Figura 2. Relación entre la longitud furcal y la masa para el barbo ibérico elaborada con el software DIMP 1.0. Fork length-mass relationship of Iberian barbel obtained using DIMP 1.0 software (http://www.gea-ecohidraulica.org/Descargas.php). 
2011). Se capturaron un total de 63 ejemplares de barbo ibérico cuyas características biométricas medias son: longitud furcal $\left(\mathrm{L}_{\mathrm{F}}\right) 16.8 \pm 2.9 \mathrm{~cm}$, masa (M) 77.0 $\pm 41.6 \mathrm{~g}$ y Factor de forma $(\mathrm{K}) 1.53$ $\pm 0.28 \mathrm{~g} / \mathrm{cm}^{3}$. En la figura 2 y en la Tabla S3 (disponible en http://www.limnetica.net/es/limnetica) se muestran dichas características con más detalle.

Los ejemplares fueron transportados hasta las instalaciones en tanques oxigenados de $100 \mathrm{~L}$. Una vez allí, fueron anestesiados con una solución de $25 \mathrm{mg} / \mathrm{L}$ de MS-222, dosis mínima recomendada para ciprínidos (Neiffer \& Stamper,
2009), para obtener sus dimensiones $\left(\mathrm{L}_{\mathrm{F}} \pm 0.1 \mathrm{~cm}\right.$ y $\mathrm{M} \pm 0.1 \mathrm{~g})$ e implantarles quirúrgicamente en la cavidad intraperitoneal la marca de telemetría PIT (TIRIS RI-TRP-WRHP, Texas Instruments, Dallas, Texas, USA: half-duplex, $23 \mathrm{~mm}$ de largo, $3.85 \mathrm{~mm}$ de diámetro y $0.6 \mathrm{~g}$ de peso -tamaños menores presentaban problemas de detección en las antenas-).

Tras la toma de medidas y el marcaje, los ejemplares se mantuvieron en una zona de aclimatación (2 estanques consecutivos de la escala de hendiduras verticales adyacente al

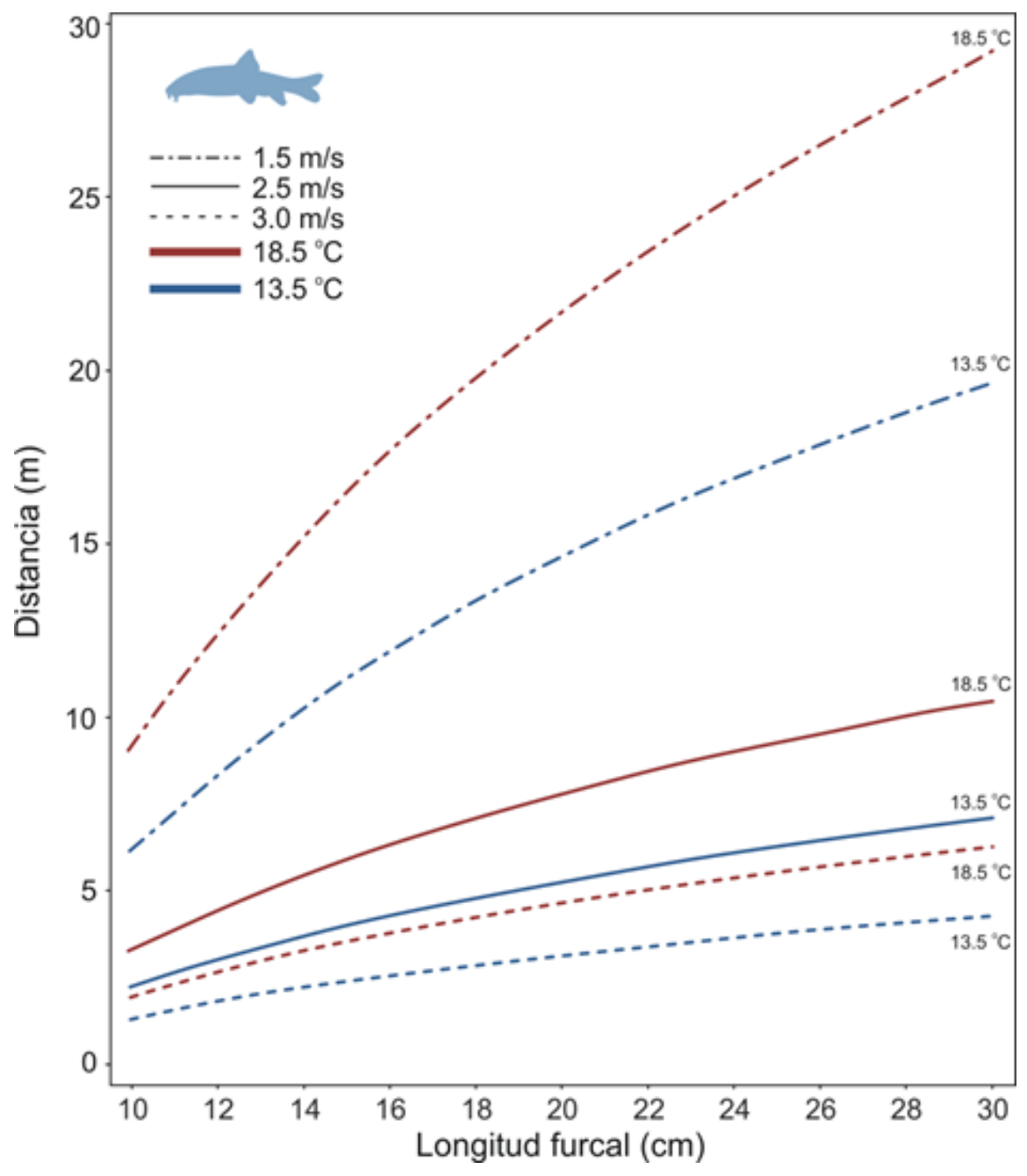

Figura 3. Modelo predictivo de la distancia recorrida $\left(\mathrm{D}_{\max }\right)$ para la longitud furcal de los individuos en función de la velocidad del flujo $(1.5 \mathrm{~m} / \mathrm{s}$-líneas discontinuas punteadas-, $2.5 \mathrm{~m} / \mathrm{s}$-líneas continuas- y $3.0 \mathrm{~m} / \mathrm{s}$-líneas discontinuas cortas- $)$ y de la temperatura del agua $\left(13.5^{\circ} \mathrm{C}\right.$-líneas azules- y $18.5^{\circ} \mathrm{C}$-líneas rojas). Prediction model of travelled distance $\left(D_{\max }\right)$ for the fork length depending on flow velocities $\left(1.5 \mathrm{~m} / \mathrm{s}\right.$-dashed-dotted line-, $2.5 \mathrm{~m} / \mathrm{s}$-solid line-and $3.0 \mathrm{~m} / \mathrm{s}$-short-dashed line-) and water temperatures $\left(13.5^{\circ} \mathrm{C}\right.$ -blue line- and $18.5^{\circ} \mathrm{C}$-red line-). 
canal, por donde circulaban $50 \mathrm{~L} / \mathrm{s}$ procedentes del río Duero) durante más de 48 h, sin que se produjese mortalidad ni se detectasen comportamientos erráticos.

\section{Ensayos}

El estudio se dividió en 2 ensayos: Ensayo 1 -36 ejemplares- con una temperatura media del agua de $18.5^{\circ} \mathrm{C}$ - del 27/05/2012 al 01/06/2012 coincidiendo con la época central de migración- $\mathrm{y}$ Ensayo 2 -27 ejemplares- con $13.5{ }^{\circ} \mathrm{C}$-del 07/05/2013 al 09/05/2013 en la época inicial de la migración-. Cada ensayo consistió en 3 experimentos con velocidades de flujo nominales de 1.5 $\mathrm{m} / \mathrm{s}$ (Exp. 1, $\mathrm{n}=36$ y Exp. 4, $\mathrm{n}=27), 2.5 \mathrm{~m} / \mathrm{s}$ (Exp. $2, \mathrm{n}=36$ y Exp. $5, \mathrm{n}=27)$ y $3.0 \mathrm{~m} / \mathrm{s}($ Exp. $3, \mathrm{n}=36$ y Exp. 6, $\mathrm{n}=27)$. En cada experimento, la muestra se dividió en 2 lotes de peces (Lote $1, \mathrm{n}=18$; Lote 2, $\mathrm{n}=18$; Lote $3, \mathrm{n}=14$ y Lote $4, \mathrm{n}=13$ ) de tamaño similar ( $p$-valor $>0.72$-ANOVA, $\alpha=0.05-)$, cada uno de los cuales fue expuesto a la gama completa de velocidades de flujo (Tabla S4 disponible en http://www.limnetica.net/es/limnetica). La duración de los experimentos fue de $4 \mathrm{~h}$ y el tiempo de descanso de cada lote entre los experimentos fue superior a $12 \mathrm{~h}$.

\section{Análisis de datos}

El estudio de datos se llevó a cabo mediante análisis de supervivencia, que incluye técnicas que permiten trabajar con líneas temporales, con datos que no presentan una distribución normal, con datos incompletos mediante la herramienta censura y con variables dependientes del tiempo. El análisis de supervivencia permite desarrollar modelos predictivos para la distancia máxima recorrida $\left(\mathrm{D}_{\max }\right)$, el tiempo de fatiga $\left(\mathrm{T}_{\mathrm{f}}\right)$ y la velocidad de nado $\left(\mathrm{U}_{\mathrm{s}}\right)$ (Haro et al., 2004; Castro-Santos, 2005; Sanz-Ronda et al., 2015). Se consideró un único intento para cada pez por experimento, utilizando aquél en el que se alcanza la mayor distancia (pues es en el que se maximiza la capacidad de nado), y se incluyeron como covariables ambientales la temperatura del agua $\left(\mathrm{T}_{\mathrm{a}}\right.$; $\left.{ }^{\circ} \mathrm{C}\right)$ y la velocidad de flujo $\left(\mathrm{U}_{\mathrm{f}} ; \mathrm{m} / \mathrm{s}\right)$ y como covariables biométricas, la longitud furcal $\left(\mathrm{L}_{\mathrm{F}} ; \mathrm{cm}\right)$, la masa (M; g) y el factor de forma (índice de Fulton;
$\mathrm{K}=100 \cdot \mathrm{M} / \mathrm{L}_{\mathrm{F}}{ }^{3}$ ). Los ascensos en los que los peces alcanzaron la última antena se consideraron como datos censurados, pues es posible que pudieran alcanzar distancias mayores, pero el sistema de registro está limitado y no es capaz de detectarlo (Hosmer \& Lemeshow, 1999).

Los modelos paramétricos (ecuaciones 5 y 6 ) se ajustaron utilizando PROC LIFEREG (SAS ${ }^{\circledR}$ University-Edition), caracterizados porque los efectos de las covariables son multiplicativos, de manera que, para un aumento de la covariable como factor aislado en 1 unidad (o el paso de un nivel a otro en el caso de covariables binarias), la variable de estudio se verá modificada en una cantidad igual a exp $(\beta)$. La selección del mejor modelo es automática mediante un proceso escalonado, que va añadiendo sucesivamente covariables (nivel de significación máximo para su entrada $-p$-valor $=0.25$ - y mínimo para formar parte del mismo $-p$-valor $=0.05-$ ). Por cada covariable añadida, se analiza si el ajuste mejora de manera estadísticamente significativa, realizando este proceso hasta que ninguna covariable mejora el modelo (Allison, 1995). Mediante el criterio de información de Akaike (AIC) se comprueba que el mejor modelo obtenido tiene el menor valor de AIC, entre todos los modelos generados.

Así, el modelo para la $\mathrm{D}_{\max }$ sigue la forma:

$$
\ln \left(D_{\max }\right)=\beta_{0}+\beta_{k} \cdot X_{k}
$$

donde $\beta$ son coeficientes de regresión y $\mathrm{X}$ son las k-covariables $\left(\mathrm{L}_{\mathrm{F}}, \mathrm{M}, \mathrm{K}, \mathrm{U}_{\mathrm{f}} \mathrm{y} \mathrm{T}_{\mathrm{a}}\right)$.

En base a las covariables que han resultado estadísticamente significativas en el modelo anterior, se analiza la relación entre $\mathrm{T}_{\mathrm{f}} \mathrm{y} \mathrm{U}_{\mathrm{s}}$, para la determinación y diferenciación de los modos de nado (prolongado: velocidad que puede mantener un pez durante $20 \mathrm{~s}-200 \mathrm{~min}$; y sprint: velocidad que puede desarrollar un pez durante menos de $20 \mathrm{~s}$ ). El análisis se realiza con un modelo lineal generalizado, aplicando la técnica del punto móvil (Castro-Santos et al., 2013), en la que mediante la iteración sucesiva de la $U_{S}$, se determina el cambio de pendiente en la propia regresión, y en consecuencia, el punto 
de cambio de natación, a través del modelo que sigue la forma:

$$
\ln \left(T_{f}\right)=\beta_{0}+\beta_{1} \cdot C_{p s}+\beta_{2} \cdot U_{s}+\beta_{3} \cdot C_{p s} \cdot U_{s}
$$

donde $\beta$ son coeficientes de regresión, $\mathrm{C}_{\mathrm{ps}}$ es una variable binaria que toma el valor 0 para el modo prolongado y 1 para el modo sprint y $\mathrm{U}_{\mathrm{s}}$ es la velocidad absoluta de nado $\left(\mathrm{U}_{\mathrm{S}}=\left(\mathrm{U}_{\mathrm{g}}+\mathrm{U}_{\mathrm{f}}\right) \cdot\left(\mathrm{L}_{\mathrm{F}} / 100\right)\right)$ en cuerpos por segundo -BL/s-.

Posteriormente, se analiza la influencia de la covariable $\mathrm{T}_{\mathrm{a}}$ en la relación $\mathrm{T}_{\mathrm{f}}-\mathrm{U}_{\mathrm{S}}$ (PROC LIFEREG -SAS ${ }^{\circledR}$ University-Edition-) estudiando su efecto principal, así como el de la interacción entre $\mathrm{T}_{\mathrm{a}}$ y $\mathrm{U}_{\mathrm{s}}$, en el $\mathrm{T}_{\mathrm{f}}$, en base a la comparación de las pendientes de las rectas de regresión.

\section{RESULTADOS}

\section{Distancia máxima}

De las covariables biométricas, la única que afectó significativamente a $\mathrm{D}_{\max }$ fue $\mathrm{L}_{\mathrm{F}}(p$-valor $<0.0061)$, con un efecto positivo, de manera que a mayor tamaño del pez, mayor distancia será capaz de recorrer, aumentando un $11 \%$ (e 0.104 $-1=0.11$ ) por cada $\mathrm{cm}$ de longitud (Tabla $1 \mathrm{y}$ Fig. 3). Así, un barbo de $18.8 \mathrm{~cm}$ (Cuartil $\mathrm{Q}_{3}$ ) recorrería un $32 \%$ más de distancia que uno de

Tabla 1. Resumen del modelo de regresión con los efectos de las covariables sobre la distancia máxima $\left(\mathrm{D}_{\max }\right)$. Los términos $\beta$ son los coeficientes de la regresión y permiten estimar cuantitativa y cualitativamente el efecto de la covariable en la distancia. SE es el error estándar. Summary of the regression model where the effect of covariables on maximum distance $\left(D_{\max }\right)$ are shown. The $\beta$ terms are regression coefficients that allow to estimate quantitatively and qualitatively the effect of the covariables on the distance. SE is the standard error.

\begin{tabular}{|c|c|c|c|c|}
\hline \multicolumn{2}{|l|}{ Parámetros } & $\beta$ & SE & $P>\chi^{2}$ \\
\hline \multicolumn{2}{|l|}{ Constante } & 2.893 & 0.451 & $<0.0001$ \\
\hline \multicolumn{2}{|l|}{ Longitud Furcal (cm) } & 0.104 & 0.038 & 0.0061 \\
\hline \multicolumn{2}{|l|}{ Masa (g) } & - & - & 0.1840 \\
\hline \multicolumn{2}{|l|}{ Factor forma $\mathrm{K}\left(\mathrm{g} / \mathrm{cm}^{3}\right)$} & - & - & 0.8099 \\
\hline$U_{f}(m / s)$ & & -1.022 & 0.072 & $<0.0001$ \\
\hline Temperatura $\left({ }^{\circ} \mathrm{C}\right)$ & 13.5 & -0.393 & 0.096 & $<0.0001$ \\
\hline Temperatura $\left({ }^{\circ} \mathrm{C}\right)$ & 18.5 & 0.000 & . & \\
\hline Weibull-shape & & 0.339 & 0.029 & \\
\hline Distribución & & Weibull & & \\
\hline N (Total) & & 108 & & \\
\hline N (Censurados) & & 19 & & \\
\hline AIC-modelo & & 136 & & \\
\hline Modelo nulo & & 260 & & \\
\hline
\end{tabular}


Tabla 2. Relación tiempo de fatiga-velocidad de nado para la covariable temperatura del agua. Se muestran los parámetros de regresión ( $\beta$ son los coeficientes; SE es el error estándar) para las dos tipologías de modo de natación, prolongado y sprint. Swim speed - Fatigue time relationship for water temperatures. Regression parameters ( $\beta$ are coefficients; SE is the standard error) for both swim modes, prolonged and sprint, are shown.

\begin{tabular}{|c|c|c|c|c|c|c|c|}
\hline \multirow{3}{*}{$\begin{array}{c}\text { Parámetros } \\
\text { regresión }\end{array}$} & \multirow{3}{*}{$\begin{array}{c}\text { Ta } \\
\text { agua } \\
\left({ }^{\circ} \mathrm{C}\right)\end{array}$} & \multicolumn{6}{|c|}{ Modo de nado } \\
\hline & & \multicolumn{3}{|c|}{ Prolongado } & \multicolumn{3}{|c|}{ Sprint } \\
\hline & & Constante & $\mathrm{U}_{\mathrm{s}}(\mathrm{BL} / \mathrm{s})$ & Escala & Constante & $\mathrm{U}_{\mathrm{s}}(\mathrm{BL} / \mathrm{s})$ & Escala \\
\hline \multirow{2}{*}{$\begin{array}{c}\beta \pm \mathrm{SE} \\
(p \text {-valor })\end{array}$} & 13.5 & $\begin{array}{c}6.624 \pm 0.865 \\
(<0.0001)\end{array}$ & $\begin{array}{c}-0.24 \pm 0.07 \\
(0.0003)\end{array}$ & 0.447 & $\begin{array}{l}3.44 \pm 0.57 \\
(<0.0001)\end{array}$ & $\begin{array}{c}-0.097 \pm 0.03 \\
(0.0012)\end{array}$ & 0.274 \\
\hline & 18.5 & $\begin{array}{c}5.076 \pm 0.965 \\
(<0.0001)\end{array}$ & $\begin{array}{c}-0.091 \pm 0.09 \\
(0.0136)\end{array}$ & 0.613 & $\begin{array}{c}3.64 \pm 0.30 \\
(<0.0001)\end{array}$ & $\begin{array}{c}-0.085 \pm 0.02 \\
(0.0001)\end{array}$ & 0.338 \\
\hline
\end{tabular}

$16.0 \mathrm{~cm}\left(\right.$ Cuartil $\left.\mathrm{Q}_{2}\right)$ manteniendo el resto de covariables constantes.

Por otro lado, las dos covariables ambientales analizadas inciden en $\mathrm{D}_{\max }$. Así, $\mathrm{U}_{\mathrm{f}}$ tiene un efecto negativo, de manera que según aumenta su valor, los individuos ascenderán menores distancias, reduciéndose un $64 \%\left(\mathrm{e}^{-1.022}-1=-0.64\right)$ por cada $\mathrm{m} / \mathrm{s}$ (Tabla 1 y Fig. 3) manteniéndose el resto de covariables constantes. Esto significa que un barbo recorre una distancia un $78 \%$ mayor frente a un flujo de $1.5 \mathrm{~m} / \mathrm{s}$ que frente al de $3.0 \mathrm{~m} / \mathrm{s}$.

Por su parte, la temperatura del agua presenta un efecto positivo en $\mathrm{D}_{\max }$. Es decir, cuando la temperatura del agua baja de $18.5^{\circ} \mathrm{C}$ a $13.5^{\circ} \mathrm{C}$, la distancia ascendida por un ejemplar de barbo se reduce un $33 \%$ (e-0,393 $-1=-0.33)$ (Tabla 2 y Fig. 3 ).

A partir del modelo de regresión, se puede predecir qué proporción de una población será capaz de superar una distancia determinada de acuerdo con Uf a la que se enfrentan y a la temperatura del agua. Analizando la figura 4 se puede establecer, por ejemplo, que a una temperatura del agua de $18.5^{\circ} \mathrm{C}$ (línea roja en Fig. 4) el $50 \%$ de una población de barbos ( $\mathrm{L}_{\mathrm{F}}$ media de $18 \mathrm{~cm}$ ) superaría distancias de 14.5, 6.1 y $3.7 \mathrm{~m}$ para $\mathrm{U}_{\mathrm{f}}$ de $1.5,2.5$ y $3.0 \mathrm{~m} / \mathrm{s}$, respectivamente. A $13.5^{\circ} \mathrm{C}$ (línea azul en Fig.4), para esas mismas velocidades, las distancias recorridas serían 11.4 $\mathrm{m}, 4.1 \mathrm{~m}$ y $2.5 \mathrm{~m}$.

\section{Relación tiempo de fatiga-velocidad de nado $\left(\mathbf{T}_{\mathrm{f}}-\mathrm{U}_{\mathrm{S}}\right)$}

La temperatura del agua influyó de forma positiva el $\mathrm{T}_{\mathrm{f}}(p$-valor $=0.002)$ y la $\mathrm{U}_{\mathrm{s}}(p$-valor $=0.028)$. Así, un descenso de $5{ }^{\circ} \mathrm{C}$ en la temperatura del agua desde $18.5^{\circ} \mathrm{C}$ a $13.5^{\circ} \mathrm{C}$, supone una reducción del $53 \%\left(\mathrm{e}^{-0,745}-1=-0.53\right)$ de $\mathrm{T}_{\mathrm{f}} \mathrm{y}$ una disminución del $10 \%\left(\mathrm{e}^{-0,109}-1=-0.10\right)$ en $\mathrm{U}_{\mathrm{s}}$.

Se observó un cambio evidente en el modo de nado (prolongado vs sprint) a velocidades de nado cercanas a $15 \mathrm{BL} / \mathrm{s}$, un límite que apenas varió en función de la temperatura (14.9 BL/s a $18.5^{\circ} \mathrm{C}$ y $15.6 \mathrm{BL} / \mathrm{s}$ a $13.5^{\circ} \mathrm{C}$ ) (Fig. 5).

En el modo sprint, ni la temperatura por sí sola, ni el efecto conjunto de la temperatura y la velocidad de nado, tienen influencia en la relación $\mathrm{T}_{\mathrm{f}}-\mathrm{U}_{\mathrm{S}}$ (Tabla 3). Sin embargo, en el modo de nado prolongado, sí influye el efecto conjunto de la temperatura con la velocidad de nado en dicha relación ( $p$-valor $=0.045)$. Así, al aumentar $\mathrm{U}_{\mathrm{S}}$ en $1 \mathrm{BL} / \mathrm{s}$ y pasar de $13.5^{\circ} \mathrm{C}$ a $18.5^{\circ} \mathrm{C}$ se produce un aumento del $2.4 \%\left(\mathrm{e}^{0.0237}-1=0.024\right)$ en $\mathrm{T}_{\mathrm{f}}$ de los barbos, debido al efecto conjunto (Tabla 3 ). 
Tabla 3. Influencia de las covariables velocidad de nado y temperatura del agua (incluyendo su interacción) en la relación tiempo de fatiga-velocidad de nado para las dos modos de natación, prolongado y sprint. Se muestran los parámetros de regresión ( $\beta$ son los coeficientes; SE es el error estándar) así como los p-valores. Influence of swim speed and water temperature covariates (including their interaction) on the relationship fatigue time-swim speed for the two swim modes, prolonged and sprint. Regression parameters and p-values are shown ( $\beta$ are the coefficients; SE is the standard error).

\begin{tabular}{|c|c|c|c|c|c|c|c|c|c|c|}
\hline \multirow{3}{*}{ Parámetro } & \multicolumn{10}{|c|}{ Modos de nado } \\
\hline & \multicolumn{5}{|c|}{ Prolongado } & \multicolumn{5}{|c|}{ Sprint } \\
\hline & Constante & $\mathrm{U}_{\mathrm{s}}(\mathrm{BL} / \mathrm{s})$ & $\mathrm{T} \mathrm{T}\left({ }^{\circ} \mathrm{C}\right)$ & $\mathrm{U}_{\mathrm{s}}{ }^{*} \mathrm{~T}$ & Escala & Constante & $\mathrm{U}_{\mathrm{s}}(\mathrm{BL} / \mathrm{s})$ & $\mathrm{T} \mathrm{T}\left({ }^{\circ} \mathrm{C}\right)$ & $\mathrm{U}_{\mathrm{s}}{ }^{*} \mathrm{~T}$ & Escala \\
\hline $\begin{array}{c}\beta \pm \mathrm{SE} \\
(p \text {-valor) }\end{array}$ & $\begin{array}{c}9.445 \pm 4.656 \\
(0.003)\end{array}$ & $\begin{array}{c}-0.529 \pm 0.392 \\
(0.012)\end{array}$ & $\begin{array}{c}-0.238 \pm 0.277 \\
(0.207)\end{array}$ & $\begin{array}{c}0.024 \pm 0.023 \\
(0.045)\end{array}$ & $0.547 \pm 0.096$ & $\begin{array}{c}6.66 \pm 2.27 \\
(0.043)\end{array}$ & $\begin{array}{c}-0.304 \pm 0.120 \\
(0.176)\end{array}$ & $\begin{array}{c}-0.163 \pm 0.129 \\
(0.391)\end{array}$ & $\begin{array}{c}0.012 \pm 0.007 \\
(0.314)\end{array}$ & $0.340 \pm 0.032$ \\
\hline
\end{tabular}

A modo de resumen, dentro del rango de valores estudiado, el barbo ibérico pudo mantener velocidades de nado en sprint ( $>15 \mathrm{BL} / \mathrm{s}$ ) durante 3-10 s, y de 17-117 s en el modo de natación prolongada $(7-15 \mathrm{BL} / \mathrm{s})$.

\section{DISCUSIÓN}

Este estudio analiza la influencia de factores ambientales y biométricos relevantes sobre el comportamiento natatorio de Luciobarbus bocagei, la especie de barbo más ampliamente distribuida en la Península y de gran similitud morfológica y biológica con el resto de congéneres ibéricos (Kotellat \& Freyhof, 2007; Doadrio et al., 2011).

La velocidad absoluta de nado en sprint que los peces pudieron desarrollar durante un tiempo de 5 $\mathrm{s}$ superó los $20 \mathrm{BL} / \mathrm{s}(3.6 \mathrm{~m} / \mathrm{s}$ para un ejemplar de $\mathrm{L}_{\mathrm{F}}=18 \mathrm{~cm}$ ), mientras que en el modo prologando, alcanzaron $10 \mathrm{BL} / \mathrm{s}(1.8 \mathrm{~m} / \mathrm{s})$ manteniéndola durante $60 \mathrm{~s}$, en las condiciones ambientales habituales durante la época de migración (Fig. 5). Estos valores superan los datos de $\mathrm{U}_{\text {crit }}$ determinados mediante respirómetros para la misma especie, entre 2-3.5 BL/s $(0.53-0.75 \mathrm{~m} / \mathrm{s})$, para una muestra de tamaños de $15-25 \mathrm{~cm}$, y una temperatura del agua de $16-21{ }^{\circ} \mathrm{C}$ (Mateus et al., 2008; Alexandre et al., 2014). $\mathrm{U}_{\text {crit }}$ se aproxima al nivel más bajo de velocidad prolongada (Sanz Ronda et al., 2015) pudiendo ser una orientación conservadora en el diseño de pasos para peces (Alexandre et al.,
2016), para zonas de transición entre flujos rápidos. Sin embargo, no parece razonable considerarla en el análisis de barreras de velocidad, pues subestima la capacidad de nado (Tudorache et al., 2007; Peake, 2008b; Castro-Santos et al., 2013).

El $\mathrm{T}_{\mathrm{f}}$ en el modo de nado en sprint no superó los $11 \mathrm{~s}-18.5^{\circ} \mathrm{C}-$ y $8 \mathrm{~s}-13.5^{\circ} \mathrm{C}$-, siendo ambos valores inferiores a los $20 \mathrm{~s}$ sugerido por Brett (1964).

De los factores ambientales considerados, $U_{f}$ fue el que más influyó en la capacidad de nado, afectando negativamente a $D_{\max }$ y a $T_{f}$, en consonancia con estudios previos (Lucas \& Frear, 1997; Tudorache et al., 2010; Sanz-Ronda et al., 2015).

El otro factor ambiental estudiado es la temperatura del agua, que Brett (1971) definió como "factor ecológico maestro" para los peces, pues regula los procesos fisiológicos (e.g., metabolismo, capacidad de nado, etc.). Los peces son organismos poiquilotermos, es decir, su temperatura interna varía considerablemente al modificarse la ambiental. La relación entre la temperatura del agua y el comportamiento de la fauna piscícola se puede representar mediante una curva con forma de campana, con una temperatura óptima que es variable entre especies (Claireaux et al., 2006). Para el barbo ibérico, el aumento de la temperatura del agua en $5{ }^{\circ} \mathrm{C}$ dentro del rango de temperatura estudiado $\left(13.5-18.5^{\circ} \mathrm{C}\right)$ tuvo un efecto positivo en la capacidad de nado. Así, la distancia que es capaz de recorrer el barbo ibérico aumenta en 1.3 veces al incrementarse en $5^{\circ} \mathrm{C}$ la temperatura. El tiempo de 
fatiga también se ve influenciado por esta diferencia de temperatura, fundamentalmente a velocidades de nado altas (>10 BL/s), siendo 1.5 veces mayor con la temperatura más alta. Lo mismo sucede con la velocidad de nado, siendo 1.1 veces mayor. En este sentido, O'Steen \& Bennett (2003) encontraron que al aumentar la temperatura del agua de $13{ }^{\circ} \mathrm{C}$ a $19{ }^{\circ} \mathrm{C}$, se incrementaba 1.6 veces la velocidad de nado del barbo común europeo (Barbus barbus Linnaeus, 1758), ciprínido potamódromo de amplia distribución en Europa.

La reducción de la capacidad de nado al disminuir la temperatura del agua puede explicarse por la combinación de factores. Al enfriarse el agua, su viscosidad dinámica aumenta, incrementándose la fuerza de arrastre que han de superar los peces (Chow, 1959), de manera que, para mantener la misma velocidad, deberán desarrollar mayor potencia (Temple \& Johnston, 1997). Además, cuando la temperatura del agua desciende, se reduce la función del sistema cardiorrespi- ratorio de los peces, principalmente la frecuencia cardiaca (Claireaux \& Audet, 2000) y la tasa de consumo de oxígeno (Alexandre \& Palstra, 2017), viéndose afectada la actividad metabólica. Sin embargo, esta relación general no siempre se ha detectado, existiendo casos en los que, o bien no hay correspondencia entre la capacidad de nado y el aumento de temperatura (Alosa pseudoharengus Wilson, $1811-8.5{ }^{\circ} \mathrm{C}-13.2{ }^{\circ} \mathrm{C}$-; Morone saxatilis Walbaum, $1792-10.7^{\circ} \mathrm{C}-24.3$ ${ }^{\circ} \mathrm{C}$-), o si existe, es negativa (Barbatula barbatula Linnaeus, 1758; Cottus gobio Linnaeus, 1758 -10 ${ }^{\circ} \mathrm{C}-20{ }^{\circ} \mathrm{C}$ en ambos casos-) (Haro et al., 2004; Tudorache et al., 2008), indicando que es necesaria más investigación a este respecto.

En el caso del barbo ibérico, el mayor rendimiento de nado dentro del rango de temperaturas estudiado sugiere que, en caso de existir un óptimo térmico, está por encima de los $18.5^{\circ} \mathrm{C}$.

En cuanto a los parámetros biométricos, es ampliamente conocido que la longitud es uno de

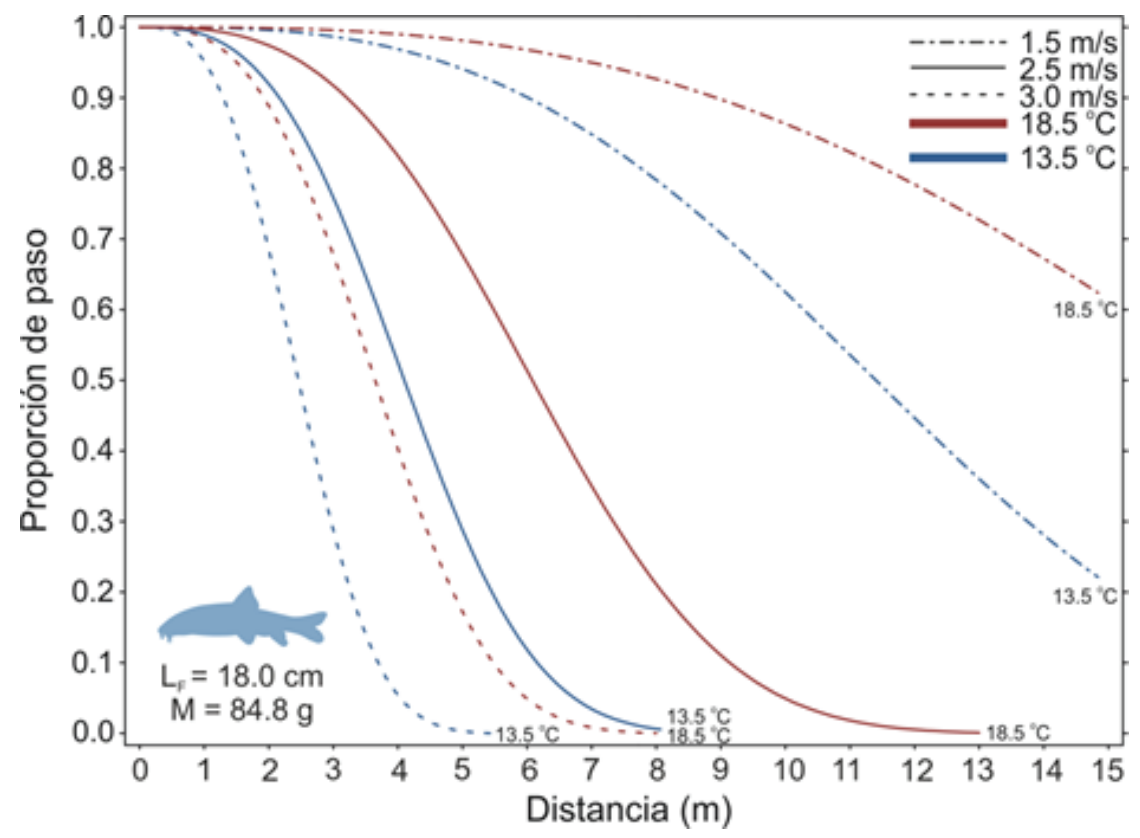

Figura 4. Curvas de supervivencia del modelo predictivo para la distancia recorrida $\left(\mathrm{D}_{\max }\right)$, mostrando la proporción de individuos que recorren una distancia dada frente a un rango de velocidades de flujo $(1.5 \mathrm{~m} / \mathrm{s}$-líneas discontinuas punteadas-, $2.5 \mathrm{~m} / \mathrm{s}$-líneas continuas- y $3.0 \mathrm{~m} / \mathrm{s}$-líneas discontinuas cortas-) y para un rango de temperaturas del agua $\left(13.5{ }^{\circ} \mathrm{C}\right.$-líneas azules- y $18.5{ }^{\circ} \mathrm{C}-$ líneas rojas-). El resto de las covariables mantienen un valor de: longitud furcal $=18 \mathrm{~cm}$ y masa $=84.8 \mathrm{~g}$. Survival curves of the ascent distance $\left(D_{\text {max }}\right)$ prediction model showing proportion passing a given distance against a range of flow velocities $(1.5 \mathrm{~m} / \mathrm{s}-$ dashed-dotted line-, $2.5 \mathrm{~m} / \mathrm{s}$-solid line- and $3.0 \mathrm{~m} / \mathrm{s}$-short-dashed line-) and with a range of water temperatures $\left(13.5^{\circ} \mathrm{C}\right.$-blue line-and $18.5^{\circ} \mathrm{C}$-red line-). The other covariables are maintained constant: fork length $=18 \mathrm{~cm}$ and mass $=84.8 \mathrm{~g}$ ). 


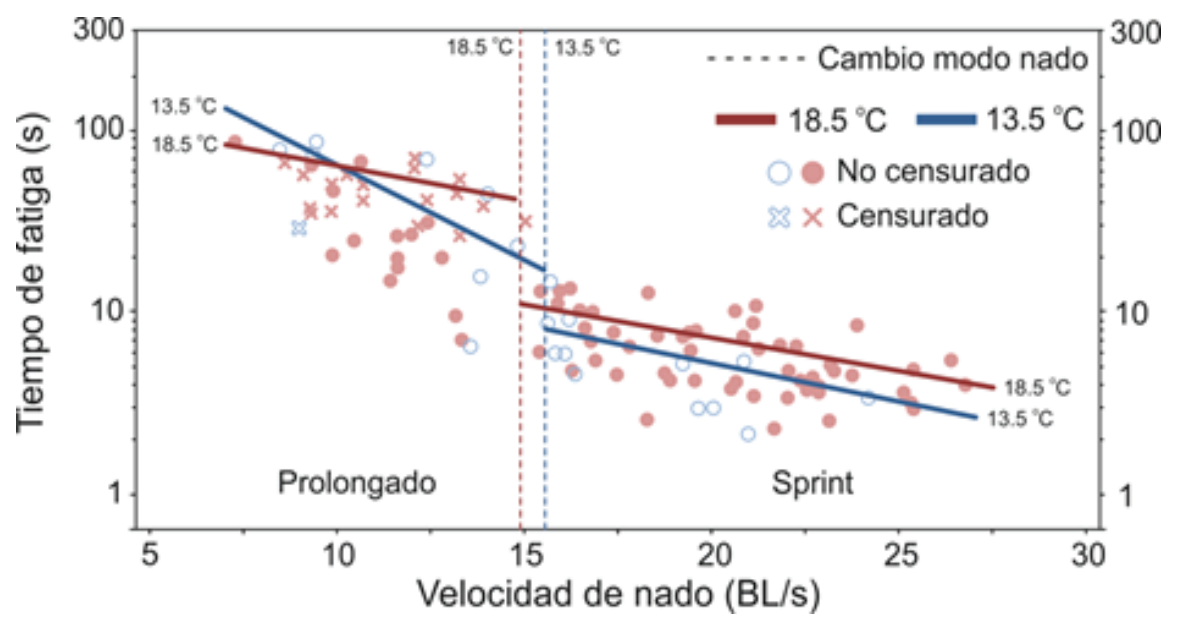

Figura 5. Relación velocidad de nado - tiempo de fatiga. Datos para temperaturas de agua de $13.5^{\circ} \mathrm{C}$ (azul) y $18.5^{\circ} \mathrm{C}$ (rojo). Las líneas verticales discontinuas indican el punto de ruptura entre los dos modos de nado (a la izquierda el modo prolongado, y a la derecha el modo sprint). Los círculos muestran los datos no censurados y las cruces los censurados. Swim speed - Fatigue time relationship for water temperatures of $13.5{ }^{\circ} \mathrm{C}$ (blue) and $18.5{ }^{\circ} \mathrm{C}$ (red). The vertical short-dashed line indicates the breakpoint selected between the two swimming modes (to the left, prolonged mode and to the right, sprint mode). Circles represent uncensored observations and crosses represent censored observations.

los factores más importantes que influyen en la capacidad de nado (Beamish, 1978; Hammer, 1995; Plaut, 2001). Este trabajo también lo confirma, en consonancia con los resultados obtenidos para el barbo ibérico en los trabajos de Mateus et al. (2008) y Sanz-Ronda et al. (2015). Esta relación positiva tiene por un lado una base biomecánica, ya que los peces más grandes presentan sistemas propulsores mayores (Webb \& Wheis, 1986), aumentando su potencia muscular (Goolish, 1989); y por otro lado una base bioquímica, pues a mayor tamaño se mejora su capacidad anaeróbica (Goolish, 1989, Ferguson et al., 1993), permitiendo desarrollar una mayor capacidad de nado. Referente a la masa, no se ha detectado una influencia significativa. La variación en la masa para una longitud dada en la misma especie aumenta considerablemente para longitudes grandes (mayores a $20 \mathrm{~cm}$ ), mientras que los ejemplares medianos y menores crecen en longitud en una proporción mucho mayor que en masa (Froese, 2006), siendo la muestra estudiada principalmente formada por ejemplares de estos dos últimos grupos (menores de $20 \mathrm{~cm}$ ).

La capacidad de nado refleja un equilibrio entre factores físicos (fuerzas gravitatorias y resistencia hidrodinámica) y metabólicos (demanda energética) (Kieffer, 2000), que se escenifica como un sacrificio entre longitud y masa (Alexandre et al., 2014). Al aumentar los niveles de reservas energéticas, que supondría incrementar la masa, se mejora el metabolismo anaeróbico, permitiendo desarrollar una mayor potencia y por tanto mejorar la capacidad de nado (Moyle \& Cech, 1996). No obstante, esta acumulación de reservas podría reducir la capacidad de natación, al tener que desplazarse una mayor masa y modificar el aspecto fusiforme de los individuos hacia un perfil hidrodinámico menos eficiente, aumentando la fuerza de arrastre (Boily \& Magnan, 2002). En este sentido, mediante el análisis del factor $\mathrm{K}$, los individuos de barbo ibérico de mayor tamaño presentan valores más bajos de $\mathrm{K}$ que los de menor talla, sugiriendo que los primeros son menos fuertes y más esbeltos e hidrodinámicos. Por ello, el parámetro $\mathrm{K}$ por sí mismo no influyó en la capacidad de nado del barbo, orientándonos hacia la búsqueda de otros factores morfométricos relacionados con la hidrodinámica del pez (Alexandre et al., 2014).

Por lo tanto, los individuos más grandes tendrán más facilidad para superar las barreras de 
velocidad que ejemplares de tallas menores, como por ejemplo machos de $7 \mathrm{~cm}$ y hembras de $18 \mathrm{~cm}$, talla mínima de maduración sexual (Lobón-Cerviá \& Fernández-Delgado, 1984; Doadrio et al., 2011). Por lo tanto, estos últimos tamaños también deberían considerarse en el diseño de sistemas de paso para peces.

A pesar de que todavía quedan algunas cuestiones importantes relativas al rendimiento de nado del barbo ibérico, la información aportada en este estudio sobre la influencia de factores como la velocidad del flujo, la temperatura del agua y las dimensiones de los individuos, puede ser utilizada como una herramienta para establecer criterios orientadores en el diseño de pasos para peces y para valorar potenciales impactos en barreras de velocidad.

Por ejemplo, los modelos desarrollados nos indican la distancia que los peces son capaces de recorrer frente a una velocidad de corriente, en función de su longitud y de la temperatura del agua. Así, en una estación de aforos del tipo V-flat (o sobre el paramento de cualquier azud, en una rampa para peces, en los vertederos de una escala, etc.), nos podríamos encontrar con velocidades medias que rondan $\operatorname{los} 3 \mathrm{~m} / \mathrm{s}$ a 10 largo de $3.5 \mathrm{~m}$ de paramento. Ello significa que en torno a un $15 \%$ de los peces con $\mathrm{L}_{\mathrm{F}}=18 \mathrm{~cm}$ serían capaces de superarla con una temperatura del agua de $13.5{ }^{\circ} \mathrm{C}$, y sobre un $65 \%$ lo conseguirían para la temperatura de $18.5{ }^{\circ} \mathrm{C}$. Estos porcentajes aumentan para $\mathrm{L}_{\mathrm{F}}$ mayores y disminuyen con LF menores.

Por lo tanto, la aplicación directa de la información aquí aportada sobre la capacidad de nado del barbo ibérico, puede ayudar a una mejor gestión de sus poblaciones y al desarrollo de programas de mejora o restauración de la conectividad longitudinal en los hábitats que ocupa esta especie.

\section{AGRADECIMIENTOS}

Esta investigación está financiada por la Junta de Castilla y León: Proyecto VA299B11-2 "Evaluación de la capacidad de nado en peces ibéricos" y enmarcada dentro del Proyecto Fishfriendly Innovative Technologies for Hydropower (FIThydro) -Programa H2020 (H2020-LCE-
2016-RES-CCS-RIA-727830). Cofinanciado por la UE-. Nuria Ramos-González (GEA-Ecohidráulica) colaboró en los trabajos de campo. Leandro Fernandes-Celestino aportó su experiencia en los tratamientos estadísticos. José María Rubio-Polo y Arturo Prieto-Blanco (CHD) promovieron las acciones que hicieron posible este proyecto. Agradecemos especialmente a Juan Carlos Romeral-de la Puente (SAVASA) por permitirnos usar sus instalaciones en Vadocondes. Cualquier uso de marcas comerciales, de productos o de empresas tiene fines descriptivos únicamente y no implica el respaldo del gobierno de EE. UU.

\section{BIBLIOGRAFÍA}

ALEXANDRE, C.M., B.R. QUINTELLA, A.F. FERREIRA, F.A. ROMÃO \& P.R. ALMEIDA. 2014. Swimming performance and ecomorphology of the Iberian barbel Luciobarbus bocagei (Steindachner, 1864) on permanent and temporary rivers. Ecology of Freshwater Fish, 23: 244-258. DOI: 10.1111/eff.12073

ALEXANDRE, C.M., R. BRANCA, B.R. QUINTELLA \& P.R. ALMEIDA. 2016. Critical swimming speed of the southern straightmouth nase Pseudochondrostoma willkommii (Steindachner, 1866), a potamodromous cyprinid from southern Europe. Limnetica, 35: 365-372.

ALEXANDRE, C. \& A. PALSTRA. 2017. Effect of short-term regulated temperature variations on the swimming economy of Atlantic salmon smolts. Conservation Physiology, 5. DOI: 10.1093/conphys/cox025

ALLISON, P.D. 1995. Survival Analysis using SAS: A Practical Guide. Sas Institute.

BEAMISH, F. 1978. Swimming capacity. In 'Fish Physiology. Vol. VII'. (Eds WS Hoar and DJ Randall.) pp. 101-187.

BOILY, P. \& P. MAGNAN. 2002. Relationship between individual variation in morphological characters and swimming costs in brook charr (Salvelinus fontinalis) and yellow perch (Perca flavescens). Journal of Experimental Biology, 205(7): 1031-1036.

BRANCO, P., J.M. SANTOS, C. KATOPODIS, A. PINHEIRO \& M.T. FERREIRA. 2013. Pool-type fishways: two different morpho- 
ecological cyprinid species facing plunging and streaming flows. PloS one, 8: e65089. DOI: 10.1371/journal.pone.0065089

BRAVO-CÓRDOBA, F.J., F.J. SANZ-RONDA, J. RUIZ-LEGAZPI, S. MAKRAKIS \& L. FERNANDES CELESTINO. 2017. "Fishway with two entrance branches: understanding its performance for potamodromous Mediterranean barbels". Fisheries Management and Ecology, FME-17-005.R1. DOI: 10.1111/fme.12260

BRETT, J. 1964. The respiratory metabolism and swimming performance of young sockeye salmon. Journal of the Fisheries Board of Canada, 21: 1183-1226. DOI: 10.1139/f64-103

BRETT, J.R. 1971. Energetic responses of salmon to temperature. A study of some thermal relations in the physiology and freshwater ecology of sockeye salmon (Oncorhynchus nerka). American Zoologist: 99-113. DOI: $10.1093 / \mathrm{icb} / 11.1 .99$

CASTRO-SANTOS, T. \& A. HARO. 2003. Quantifying migratory delay: a new application of survival analysis methods. Canadian Journal of Fisheries and Aquatic Sciences, 60: 986-996. DOI: 10.1139/f03-086

CASTRO-SANTOS, T. 2004. Quantifying the combined effects of attempt rate and swimming capacity on passage through velocity barriers. Canadian Journal of Fisheries and Aquatic Sciences, 61: 1602-1615. DOI: 10.1139/f04-094

CASTRO-SANTOS, T. 2005. Optimal swim speeds for traversing velocity barriers: an analysis of volitional high-speed swimming behavior of migratory fishes. The Journal of experimental biology, 208: 421-432. DOI: 10.1242/jeb.01380

CASTRO-SANTOS, T., F.J. SANZ-RONDA \& J. RUIZ-LEGAZPI. 2013. Breaking the speed limit - comparative sprinting performance of brook trout (Salvelinus fontinalis) and brown trout (Salmo trutta). Canadian Journal of Fisheries and Aquatic Sciences, 70: 280-293. DOI: $10.1139 /$ cjfas-2012-0186

CHD. 2015. Plan Hidrológico de la parte española de la demarcación hidrográfica del Duero (2015-2021). Anejo 7: inventario de presiones. Confederación Hidrográfica del Duero. Valladolid. España.
CLAIREAUX, G. \& C. AUDET. 2000. Seasonal changes in the hypo-osmoregulatory ability of brook charr: the role of environmental factors. Journal of fish biology, 56: 347-373. DOI: 10.1111/j.1095-8649.2000.tb02111.x

CLAIREAUX, G., C. COUTURIER \& A.L. GROISON. 2006. Effect of temperature on maximum swimming speed and cost of transport in juvenile European sea bass (Dicentrarchus labrax). The Journal of experimental biology, 209: 3420-3428. DOI: $10.1242 / \mathrm{jeb} .02346$

COOKE, S.J., C. PAUKERT \& Z. HOGAN. 2012 b. Endangered river fish: factors hindering conservation and restoration. Endangered Species Research, 17: 179-191. DOI: 10.3354/esr00426

DOADRIO, I., S. PEREA, P. GARZÓN-HEYDT \& J.L. GONZÁLEZ. 2011. Ictiofauna continental española. Bases para su seguimiento. DG Medio Natural y Política Forestal. MARM. Madrid. España.

FERGUSON, R.A., J.D. KIEFFER \& B.L. TUFTS. 1993. The effects of body size on the acid-base and metabolite status in the white muscle of rainbow trout before and after exhaustive exercise. Journal of Experimental Biology, 180: 195-207.

GOOLISH, E.M. 1989. The scaling of aerobic and anaerobic muscle power in rainbow trout (Salmo gairdneri). Journal of Experimental Biology, 147: 493-505.

HAMMER, C. 1995. Fatigue and exercise tests with fish. Comparative Biochemistry and Physiology Part A: Physiology, 112: 1-20. DOI: 10.1016/0300-9629(95)00060-K

HARO, A., T. CASTRO-SANTOS, J. NOREIKA \& M. ODEH. 2004. Swimming performance of upstream migrant fishes in open-channel flow: a new approach to predicting passage through velocity barriers. Canadian Journal of Fisheries and Aquatic Sciences, 61: 1590-1601. DOI: 10.1139/f04-093

HOSMER JR, D.W. \& S. LEMESHOW. 1999. Applied survival analysis: Regression modelling of time to event data (1999).

KIEFFER, J.D. 2000. Limits to exhaustive exercise in fish. Comparative Biochemistry and Physiology Part A: Molecular \& Integrative Physiology, 126: 161-179. DOI: 10.1016/S1095-6433 (00)00202-6 
KOTTELAT, M. \& J. FREYHOF. 2007. Handbook of European Freshwater Fishes. Publications Kottelat. Cornol. Switzerland.

LOBÓN-CERVIÁ, J. \& C. FERNÁNDEZ-DELGADO. 1984. On the biology of the barbel (Barbus barbus bocagei) in the Jarama River. Folia Zoologica, 33: 371-384.

LUCAS, M. \& P. FREAR. 1997. Effects of a flow-gauging weir on the migratory behaviour of adult barbel, a riverine cyprinid. Journal of fish biology, 50: 382-396. DOI: 10.1111/j.10958649.1997.tb01366.x

LUCAS, M. \& E. BARAS. 2001. Migration of Freshwater Fishes Blackwell Science Ltd. London 420.

MATEUS, C., B.R. QUINTELLA \& P.R. ALMEIDA. 2008. The critical swimming speed of Iberian barbel Barbus bocagei in relation to size and sex. Journal of fish biology, 73: 1783-1789. DOI: 10.1111/j.1095-8649. 2008.02023.x

MORITA, K. \& S. YAMAMOTO. 2002. Effects of habitat fragmentation by damming on the persistence of stream-dwelling charr populations. Conservation Biology, 16: 1318-1323. DOI: 10.1046/j.1523-1739.2002.01476.x

MOYLE, P.B. \& J.J. CECH. 1996. Fishes: An Introduction to Ichthyology. Prentice Hall Englewood Cliffs. NJ. USA.

O'STEEN, S. \& A.F. BENNETT. 2003. Thermal acclimation effects differ between voluntary, maximum, and critical swimming velocities in two cyprinid fishes. Physiological and Biochemical Zoology: PBZ, 76: 484-496.

PAVLOV, D., A. LUPANDIN \& V. KOSTIN. 2002. Downstream Migration of Fish through Dames of Hydroelectric Power Plants. Oak Ridge National Laboratory. Oak Ridge. USA.

PEAKE, S. 2008a. Gait transition speed as an alternate measure of maximum aerobic capacity in fishes. Journal of fish biology, 72: 645-655. DOI: 10.1111/j.1095-8649.2007. 01753.x

PEAKE, S.J. 2008b. Swimming performance and behavior of fish species endemic to Newfoundland and Labrador: a literature review for the purpose of establishing design and water velocity criteria for fishways and culverts. Canadian Manuscript Report of
Fisheries and Aquatic Sciences, Canadian Journal of Fisheries and Aquatic Sciences, 2843: v, 52.

PLAUT, I. 2001. Critical swimming speed: its ecological relevance. Comparative Biochemistry and Physiology Part A: Molecular \& Integrative Physiology, 131: 41-50. DOI: 10.1016/S1095-6433(01)00462-7

ROMÃO, F., B. QUINTELLA, T. PEREIRA \& P. ALMEIDA. 2012. Swimming performance of two Iberian cyprinids: the Tagus nase Pseudochondrostoma polylepis (Steindachner, 1864) and the bordallo Squalius carolitertii (Doadrio, 1988). Journal of Applied Ichthyology, 28: 26-30. DOI: 10.1111/j.1439-0426. 2011.01882.x

SANTOS, J.M., A. SILVA, C. KATOPODIS, P. PINHEIRO, A. PINHEIRO, J. BOCHECHAS \& M.T. FERREIRA. 2012. Ecohydraulics of pool-type fishways: getting past the barriers. Ecological Engineering, 48: 38-50. DOI: 10.1016/j.ecoleng.2011.03.006

SANZ-RONDA, F.J., J. RUIZ-LEGAZPI, F.J. BRAVO-CÓRDOBA, S. MAKRAKIS \& T. CASTRO-SANTOS. 2015. Sprinting performance of two Iberian fish: Luciobarbus bocagei and Pseudochondrostoma duriense in an open channel flume. Ecological Engineering, 83: 61-70. DOI: 10.1016/j.ecoleng.2015.05.033

SILVA, A.T., C. KATOPODIS, J.M. SANTOS, M.T. FERREIRA \& A.N. PINHEIRO. 2012. Cyprinid swimming behaviour in response to turbulent flow. Ecological Engineering, 44: 314-328. DOI: 10.1016/j.ecoleng. 2012. 04.015

TE CHOW, V. 1959. Open Channel Hydraulics. McGraw-Hill Book Company, Inc. New York. USA.

TEMPLE, G.K. \& I.A. JOHNSTON. 1997. The thermal dependence of fast-start performance in fish. Journal of thermal biology, 22: 391-401. DOI: 10.1016/S0306-4565(97)00058-2

TUDORACHE, C., P. VIAENEN, R. BLUST \& G. DE BOECK. 2007. Longer flumes increase critical swimming speeds by increasing burst-glide swimming duration in carp Cyprinus carpio, L. Journal of fish biology, 71: 1630-1638. DOI: 10.1111/j.1095-8649. 2007.01620.x 
TUDORACHE, C., P. VIAENE, R. BLUST, H. VEREECKEN \& G. DE BOECK. 2008. A comparison of swimming capacity and energy use in seven European freshwater fish species. Ecology of Freshwater Fish, 17: 284-291. DOI: $10.1111 / \mathrm{j} .1600-0633.2007 .00280 . \mathrm{x}$

TUDORACHE, C., R. O'KEEFE \& T. BENFEY. 2010. The effect of temperature and ammonia exposure on swimming performance of brook charr (Salvelinus fontinalis). Comparative Biochemistry and Physiology Part A: Molecular \& Integrative Physiology, 156: 523-528. DOI: 10.1016/j.cbpa.2010.04.010

VIDELER, J. \& C. WARDLE. 1991. Fish swimming stride by stride: speed limits and endurance. Reviews in Fish Biology and Fisheries, 1: 23-40.

VIDELER, J.J. 1993. Fish Swimming. Chapman \& Hall. London. UK.

WARDLE, C. 1975. Limit of fish swimming speed. Nature, 255: 725-727. DOI: 10.1038/ 255725a 0

WEAVER, C.R. 1963. Influence of water velocity upon orientation and performance of adult migrating salmonids. Fishery Bulletin, 63: 97-121.

WEBB, P.W. \& D. WEIHS. 1986. Functional locomotor morphology of early life history stages of fishes. Transactions of the American Fisheries Society, 115: 115-127. 